\title{
Introdução ao GenVirtual: uma interface musical com realidade aumentada para apoiar o "fazer musical" de pessoas com deficiên- cia motora e cognitiva
}

Introducing GenVirtual: an augmented reality musical interface to support the "music-making" for people with motor and cognitive disability

\author{
Ana Grasielle Dionísio Corrêa \\ Laboratório de Sistemas Integráveis da Escola Politécnica \\ da Universidade de São Paulo (LSI-EPUSP). \\ anagrasi@1si.usp.br
}

Marilena do Nascimento

Associação de Assistência à Crianças Deficientes (AACD) musicoreab-marilena@aacd.org.br
Irene Karaguilla Ficheman

Laboratório de Sistemas Integráveis da Escola Politécnica da Universidade de São Paulo (LSI-EPUSP).

irene@1si.usp.br

\section{Roseli de Deus Lopes}

Laboratório de Sistemas Integráveis da Escola Politécnica da Universidade de São Paulo (LSI-EPUSP).

roseli@1si.usp.br

\begin{abstract}
Resumo Este artigo apresenta a pesquisa e o desenvolvimento do GenVirtual, uma interface musical com realidade aumentada, para apoiar o "fazer musical" de crianças com deficiência motora e cognitiva. O GenVirtual possibilita adicionar, no mundo real, objetos virtuais sonoros que emitem sons de diversos instrumentos musicais como os de corda, sopro e percussão. Os objetos virtuais são manipulados naturalmente, com as mãos, sem uso de aparatos convencionais de interação. Durante a fase de desenvolvimento, o GenVirtual foi avaliado por um grupo composto por 11 especialistas em musicoterapia com vistas a validar o conjunto de requisitos relacionados ao "fazer musical" pedagógico e terapêutico. Ao final do desenvolvimento, o GenVirtual foi avaliado por um grupo de 5 crianças com déficits motores e cognitivos em sessões de musicoterapia. Os resultados mostraram que o GenVirtual possibilitou apoiar as abordagens utilizadas no "fazer musical". Além disso, melhorou o desempenho e o estímulo da ação das crianças, características estas que contribuem para a motivação durante o "fazer musical.
\end{abstract}

Palavras-Chave: realidade aumentada, fazer musical, musicoterapia.

\begin{abstract}
This paper presents the research and development of GenVirtual, an augmented reality musical interface to support the "music-making" for children with physical disabilities and cognitive. GenVirtual enables adding in the real world, virtual objects that emit audible sounds of various musical instruments like the stringed, wind and percussion. Virtual objects are handled naturally by hand, without the use of conventional apparatuses interaction. During the development phase, GenVirtual was evaluated by a group of 11 experts in music in order to validate the set of requirements related to pedagogical and therapeutic "make music". At the end of development, GenVirtual was evaluated by a group of 5 children with cognitive and motor deficits in music therapy sessions. Results showed that GenVirtual possible approaches used in the "musicmaking". Moreover, improved performance and stimulating action of children, these characteristics that contribute to motivation during the "music-making".
\end{abstract}

Keywords: augmented reality, music-making, music therapy. 


\section{Introdução}

O "fazer musical" é uma forma de explorar e produzir sons e silêncio, explorar materiais sonoros diversos e participar de brincadeiras e jogos musicais $[1,2]$. Os benefícios do "fazer musical" para o ser humano são muitos [3,4]: ampliam a capacidade de cognição, alimentam mudanças no potencial perceptivo e possibilitam acessar a parte do cérebro que funciona criativa e intuitivamente, favorecendo novas formas de sentir, pensar e expressar e conhecer a si mesmo.

O "fazer musical" vem sendo utilizado tanto pedagogicamente $[5,6]$, como base para o ensino de teoria e práticas musicais, como também terapeuticamente $[7,8,9]$ para melhorar aspectos físicos, cognitivos, emocionais e sociais dos indivíduos. Os estímulos musicais podem alterar a respiração, circulação sanguínea, digestão, oxigenação e dinamismo nervoso e humoral [10]. Também estimulam a energia muscular, reduzem a fadiga e favorecem o tônus muscular [11]. Podem baixar o limiar da dor e se constituir como um importante recurso contra o medo e a ansiedade [12]. Podem aumentar a atenção e a concentração e estimular a memória [13, 14]. Podem também estimular atividades lúdicas que diminuem a inatividade, servindo como linha de fuga diante das rotinas impostas pelos centros de tratamento, fatores geradores de ansiedade, sofrimento e depressão [15, 16, 17].

Para algumas pessoas com deficiência, seja ela motora ou cognitiva, faz-se necessário viabilizar o "fazer musical" por meio de técnicas ou métodos adaptativos que facilitam a interação com os instrumentos e brincadeiras musicais $[18,19,20]$. Apesar do avanço na criação e confecção dessas adaptações, uma pesquisa de campo feita na fase inicial desta pesquisa, constatou alguns contrapontos: algumas adaptações são feitas sob encomenda e, portanto, em pequenas quantidades para quem os solicita (professor/terapeuta); algumas vezes, um recurso adaptador de prática instrumental atende apenas às necessidades de uma determinada incapacidade física, sendo que para outras, este mesmo recurso pode parecer inapropriado; para alguns indivíduos, pode ser interessante colocar em prática algumas orientações recebidas pelo especialista (professor/terapeuta) em ambiente domiciliar, entretanto, a situação econômica de algumas pessoas associada ao elevado preço de alguns instrumentos musicais adaptados, dificulta ou inviabiliza a continuidade das atividades musicais em ambiente domiciliar.

Por outro lado, o número de computadores e acesso à Internet nas escolas e nos lares brasileiros vem crescendo rapidamente [21, 22]. Além de promover o acesso à informação e comunicação, a inclusão digital, tem se tornado uma importante aliada do ensino e aprendizagem $[23,24]$, do treinamento [25] e da simulação [26]. Dessa forma, diversas tecnologias computacionais, com recursos para pessoas com deficiência cognitiva e física, vêm tornando possível o "fazer musical" terapêutico e educacional $[27,28,29]$.

Nesta pesquisa, buscou-se investigar se seria possível conceber uma interface musical com realidade aumentada, de baixo custo, capaz de apoiar e ampliar as abordagens do "fazer musical" de pessoas com deficiência. A metodologia da pesquisa seguiu a estratégia exploratória, de natureza tecnológica aplicada, tendo como objetivo a geração de um produto (GenVirtual) com finalidades imediatas, capaz de viabilizar testes e estudos em situações reais de uso. Colaboraram com esta pesquisa, especialistas em música, musicoterapeutas e crianças com déficits motores e cognitivos em tratamento no setor de musicoterapia da Associação de Assistência à Criança com Deficiência (AACD). Os dados foram coletados por meio de questionários, observações e entrevistas semiestruturadas, e submetidos à análise qualitativa e quantitativa. Os resultados apontaram o GenVirtual como um instrumento facilitador e motivador em atividades de "fazer musical" de crianças com deficiência cognitiva (com déficits de atenção, concentração, memória e planejamento) e motora (membros superiores com amplitude de movimentos limitada).

\section{Adaptações para o "fazer musical" de Pessoas com Deficiência}

O "fazer musical" $[18,19]$ pode envolver atividades de: exploração, expressão e produção de sons e silêncios com uso de materiais sonoros diversos; interpretação de músicas e canções diversas; participação em brincadeiras e jogos cantados e rítmicos. De acordo com [1,2], o "fazer musical" é uma das formas de relação do ser humano com o sonoro, forma de organização da percepção, de manifestação consciente, de realização complexa, de pensamento, de comunicação e expressão e do jogo; é um dos sistemas de aprendizado que pode atualizar o modo de ser humano (que envolve o perceber, sentir, pensar, comunicar, interagir, organizar, criar e repetir). Para tanto, se faz necessário o uso de instrumentos musicais convencionais ou tecnologias computacionais musicais.

O fazer musical, muitas vezes, requer a utilização de técnicas compensatórias ou adaptativas, quando dirigidas especificamente às pessoas com deficiência física. Atualmente, podem ser encontradas diversas adaptações nos instrumentos musicais $[18,19]$. Um exemplo é um teclado eletrônico adaptado, denominado de "Big Keyboard" [18]. O teclado é constituído de madeira leve e alumínio, com teclas ampliadas em $5,5 \mathrm{~cm}$ em suas dimensões. 
Dessa forma, uma pessoa com uma mão hipotônica, sem destreza dos dedos, por exemplo, ao invés de digitar, pode utilizar as mãos fechadas em forma de punho para percutir as teclas do piano. Outro exemplo é um suporte regulável à altura para sustentação do pandeiro. Este recurso facilita a utilização do instrumento por pessoas que não possuem um dos braços ou que tenham dificuldades em segurar ou manipular o instrumento. Há também suportes para triângulos e outros instrumentos de percussão. Esse suporte facilita a utilização de baquetas, mesmo quando a pessoa se encontra sentada em banco baixo ou no chão.

Além das adaptações para os instrumentos musicais, existem também softwares específicos que possibilitam o acesso ao computador por meio de comandos simples [27], adaptações no teclado ou mouse, associados a programas musicais [29], de forma a permitir a escrita musical ou a programação e gravação de arranjos musicais.

De acordo com a referência [19], havendo múltiplas possibilidades de adaptações, haverá múltiplas possibilidades de se realizar o "fazer musical", o que certamente favorece as pessoas com deficiência motora e que desejam estar em contato com a música. Mesmo assim, ainda existem casos em que mesmo estas adaptações não são suficientes para a inserção no universo musical. É o caso de indivíduos com doenças neuromusculares, em que é fundamental que os terapeutas trabalhem com perdas funcionais e economia física energética. Nestes casos, algumas atividades musicais não são recomendadas, pois podem favorecer a fadiga e o desequilíbrio muscular devido ao excesso de esforço físico e repetitivo que os instrumentos musicais exigem.

\section{Interfaces Musicais}

Diversas tecnologias vêm sendo utilizadas no design de interfaces musicais. Muitas delas favorecem a inclusão de pessoas com deficiência motora em atividades de educação e expressão musical e musicoterapia. Interfaces não convencionais como, por exemplo, as Interfaces Tangíveis, têm proporcionado uma interação mais natural, amenizando algumas dificuldades tais como: manipulação de instrumentos musicais convencionais e aparatos convencionais como mouse e teclado.

Interfaces Tangíveis, do inglês Tangible User Interfaces (TUI), podem ser definidas como uma interface entre o homem e o computador, onde objetos físicos são utilizados para representar e controlar informações digitais [30]. Enquanto que, nas Interfaces Gráficas Convencionais, do inglês Graphical User Interface (GUI), os usuários usam periféricos convencionais como teclado e mou- se para interagir com o computador, nas TUIs os usuários manipulam objetos físicos do mundo real para alcançar os mesmos resultados [31].

O potencial atrativo de interação das TUIs tem proporcionado o surgimento de diversas interfaces interativas para criação e expressão musical. Interfaces Musicais Tangíveis ou, simplesmente, TUIs Musicais buscam prover meios atrativos e intuitivos de manipular informações musicais [32]. Esta característica das TUIs tem proporcionado a inclusão de pessoas com deficiência que não conseguem interagir com os instrumentos musicais convencionais [33].

Várias tecnologias são usadas na concepção de TUIs Musicais como, por exemplo, sensores óticos, emissores de luz como LEDS coloridos e infravermelhos, telas sensíveis ao toque, entre outras. Por meio de sensores óticos (câmeras), por exemplo, é possível rastrear objetos do mundo físico e transformar os dados capturados em informações áudio-visuais. O campo de pesquisa é vasto [32,33,34,35,36,37,38,39] e, normalmente, requer a colaboração de uma equipe multidisciplinar como designer, engenheiros e músicos. A seguir são apresentados alguns exemplos de TUIs Musicais que têm alguma semelhança com o projeto desenvolvido.

\subsection{Trabalhos Correlatos}

O AudioCube [34] é constituído de objetos cúbicos sonoros fabricados de um material plástico translúcido contendo sensores óticos e emissores de luz (LEDs coloridos e infravermelho). Cada um destes objetos pode ser disposto numa superfície de forma independente. As propriedades sonoras destes objetos podem ser alteradas de acordo com as relações espaciais entre eles. Em outros casos, os objetos podem ser combinados para criar uma estrutura musical maior. Cada cubo contém uma bateria capaz de processar os sons em tempo real. Os componentes eletrônicos podem ser configurados por meio de um cabo conectado a um computador onde um software é usado para baixar novos algoritmos de processamento de som para os cubos.

O ReacTable $[35,36]$ é uma mesa interativa que possibilita manipular objetos tangíveis que emitem sons. Assim que os objetos são colocados sobre a superfície da mesa, ficam iluminados e começam a interagir com os outros objetos, de acordo com suas posições e fator de proximidade. Existem diversos objetos com funções diferentes: geradores de som, samplers e loops, filtros e efeitos, osciladores de baixa frequência ou pulsos. Todos os objetos têm funções e comportamentos diferentes e todos eles podem ser combinados entre si. Um projetor, situado abaixo da superfície da mesa, fornece um feedback visual do estado e das principais características dos 
sons produzidos pelos objetos. Uma câmera também situada abaixo da superfície analisa, continuamente, a localização, posição e orientação de objetos físicos que são distribuídos na superfície da mesa. A atração desse instrumento está na sua estética visual, pois a mistura do azul translúcido da mesa, com o jogo de cores dos objetos tangíveis provoca um fascínio à visão, proporcionando uma fusão de linguagens entre o visual e o musical. $\mathrm{O}$ inovador desse sistema reside na possibilidade de várias pessoas interagirem simultaneamente, o que estimula as novas tendências embarcadas pela criação coletiva. $\mathrm{O}$ ReacTable foi adaptado recentemente aos tablets e smart phones tanto em iOS como em Androids com o nome de Reactable Mobile [37].

O ReacTable foi usado por pesquisadores da Universidade Pompeu Fabra, na Espanha, para investigar se seu uso colaborativo ajuda a melhorar as habilidades de interação social de crianças com autismo [38]. Os pesquisadores constataram, por meio de análise quantitativa e qualitativa, que o ReacTable possibilitou, durante as sessões, um aumento da interação social propiciada pela criação coletiva de arranjos musicais e brincadeiras recíprocas entre as crianças.

O d-touch [39] é um sistema similar ao ReacTable com a vantagem de se apresentar como uma solução economicamente menos dispendiosa. Os objetos tangíveis são blocos retangulares feitos de papel. Cada bloco é marcado com um símbolo sob a forma de uma nota musical. Os blocos são posicionados sobre uma folha de papel A4 com o desenho de uma pauta musical. Uma webcam captura imagens dos símbolos dos blocos sobre uma pauta, calcula sua posição e orientação em relação à pauta e o sistema emite os sons correspondentes às notas musicais. A altura (frequência) da nota musical depende da posição vertical do objeto sobre a pauta, enquanto que sua posição horizontal mostra o momento em que a nota deve ser tocada, como ocorre no método convencional de escrita musical. Os objetos (notas musicais) estão disponíveis em diferentes tipos, cada um representando uma duração diferente (semibreve, mínima, semínima etc). Quando o final da pauta é atingido, o sistema regressa ao início da pauta. Duas ou mais notas musicais com duração semelhante podem se dispostas na pauta formando um acorde musical.

O d-touch foi avaliado informalmente por quarto educadores e dois especialistas em musicoterapia. Os avaliadores indicaram o d-touch para o ensino musical utilizando o método Suzuki [40]. Este método baseia-se na aprendizagem natural da fala, ou seja, no aprendizado da língua materna, que se desenvolve naturalmente por meio da repetição: o recém nascido ouve sua mãe e demais familiares a falar e, naturalmente, a criança se motiva a aumentar seu repertório e formar pequenas frases. O mesmo processo pode ocorrer com uso do d-touch: o ambiente musical, conduzido por um especialista, poderá levar o aluno a dominar a linguagem musical, levando-o a adquirir habilidades para a música.

O Music Table [41] é um sistema de realidade aumentada para composição musical. A realidade aumentada possibilita capturar informações do mundo real do usuário e transformá-las em objetos virtuais que podem ser inseridas no mundo real e mostradas ao usuário por meio de algum dispositivo tecnológico de visualização [42]. No Music Table, o usuário organiza cartões feitos de papel sobre uma superfície para criar frases musicais. Cada cartão representa uma nota musical. A posição horizontal e vertical dos cartões sobre uma superfície marcam o tempo e a altura das notas musicais respectivamente. A posição dos cartões na mesa é controlada por uma câmara ligada a um computador na qual as imagens capturadas pela câmara são exibidas para o usuário por meio de um monitor de vídeo. O software gera imagens de criaturas animadas para fornecer feedback para o usuário. A animação dessas criaturas é feita à medida que o volume das notas é alterado.

Dentre as principais vantagens dos sistemas de realidade aumentada, destacam-se [43]: a) possibilidade de conceber aplicações mais atrativas com representações visuais, auditivas e sinestésicas que motivam o usuário; b) ambas as mãos pode ser utilizada para a interação com os objetos virtuais, pois o usuário não precisa segurar um dispositivo tecnológico; c) vários objetos virtuais podem ser manipulados simultaneamente; d) possibilidade de conceber sistemas interativos de baixo custo.

O GenVirtual foi desenvolvido com recursos de realidade aumentada acrescido de recursos sonoros. A seguir são apresentados os detalhes de implementação e funcionamento do sistema.

\section{Desenvolvimento do GenVirtual}

O GenVirtual é uma interface musical concebida com realidade aumentada. Durante seu desenvolvimento, foram testadas as bibliotecas ARToolkit, FLARToolkit e JARToolkit. A biblioteca ARToolkit apresentou maior eficiência em relação ao número simultâneo de cartões marcadores possíveis de serem utilizados sem que houvesse confusão no momento de reconhecimento dos símbolos cadastrados. Como as funções e rotinas da biblioteca ARToolkit estão implementadas na linguagem de programação $\mathrm{C}$, foi necessário identificar bibliotecas de som compatível com essa linguagem para integração dos recursos sonoros. Foram feitos estudos com a biblioteca de som do Windows (API Win32) e a biblioteca OpenAL e ambas passaram a ser utilizadas. Para desenvolvimento de todo o sistema, foram utilizadas seis bibliotecas: 
Software Development Kit (SDK) VideoLib: biblioteca usada para manipulação dos parâmetros e fluxos de vídeos.

- ARToolkit: biblioteca de realidade aumentada que realiza rastreamento ótico usado para calibração da webcam e detecção de marcadores que fazem parte do cenário do sistema.

- Microsoft Foundation Class Library (MFC): biblioteca usada para gerenciamento da interface gráfica com o usuário. Possui uma coleção de classes usadas em programação orientada a objetos que podem ser usadas para construir interfaces como, por exemplo, para impressão de textos na tela e carregamento de imagens.

- OpenAL (Open Audio Library): biblioteca multiplataforma desenvolvida para manipular arquivos de áudio multicanal tridimensional.

- API Win32: biblioteca do Windows composta de rotinas para a manipulação de mensagens MIDI, do inglês Musical Instrument Digital Interface, que podem ser enviadas ao sintetizador MIDI disponível na placa de som do computador. Estas mensagens especificam a nota musical e suas características (altura, velocidade, intensidade e timbre).

- OpenGL: biblioteca para manipulação de rotinas gráficas e de modelagem, bidimensional (2D) e tridimensional (3D).

\subsection{Funcionamento Básico}

Para o funcionamento do GenVirtual são necessários: uma câmera equipada com tripé; o software de realidade aumentada GenVirtual; cartões musicais para interação; e uma tela para exibir os objetos 3D. A Figura 1 mostra os componentes e layout de uso do GenVirtual.

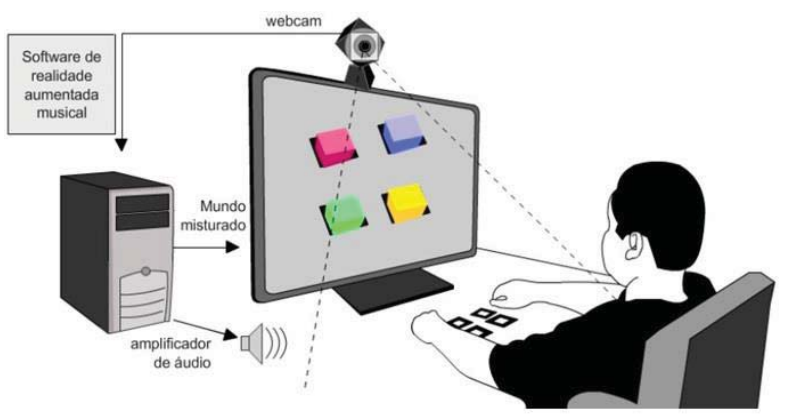

Figura 1: Componentes para uso do GenVirtual

Os cartões marcadores são formados por símbolos musicais impressos em suas faces. Os símbolos musicais se dividem em notas musicais e instrumentos musicais. As notas musicais são representadas por notações musicais, como por exemplo, o uso das palavras (Dó, Ré, Mi, Fá, Sol, Lá e Si), e os instrumentos musicais pelo desenho do instrumento (Figura 2).

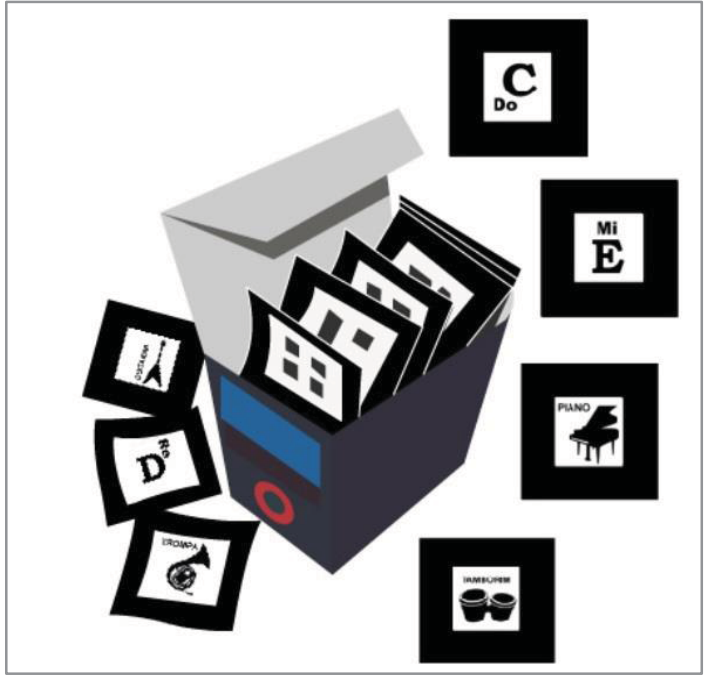

Figura 2: Cartões musicais do GenVirtual.

Os desenhos dos símbolos musicais foram um dos requisitos levantados com os especialistas durante o desenvolvimento. Segundo os especialistas em música, os desenhos nos cartões facilitam o planejamento das atividades musicais. Cada cartão musical origina um objeto virtual sonoro (cubo virtual). Para visualizar estes objetos, o usuário deve posicionar os cartões musicais no campo de visão da webcam. Para percutir os sons das notas musicais, o usuário deve "tocar" nos objetos virtuais, ou seja, obstruir o cartão musical no campo de visão da webcam (Figura 3). Os objetos virtuais podem variar em forma, tamanho e cor dependendo do desafio desejado para a atividade musical. Dependendo da tarefa planejada, os objetos virtuais disparam eventos sonoros e visuais diferenciados.

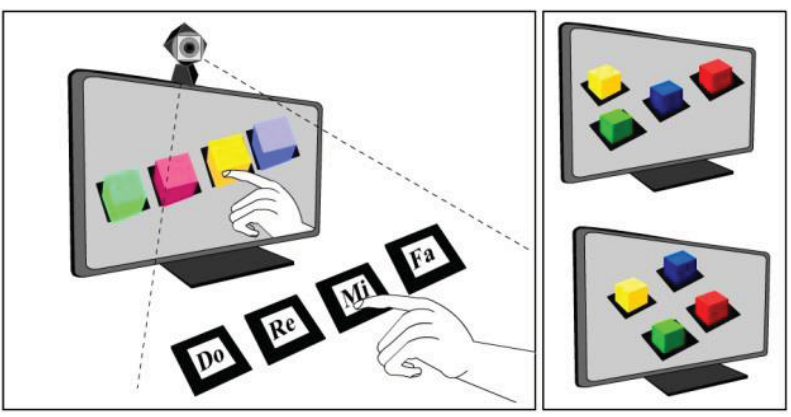

Figura 3: Posicionamento dos cartões musicais

O usuário pode efetuar movimentos desde um simples ralar dos dedos (Figura 4.a) e 4.b) até uma batida com as mãos fechadas em forma de punho sobre os cartões marcadores (Figura 4.c). Essa é uma das características do GenVirtual, independentemente do tipo de atrofia nas mãos, a interação com o sistema ocorre sempre da mesma forma e sem adaptações, mesmo para àqueles que não possuem deficiência. Comparamos este recurso com o 
teclado "Big Keyboard" [18] apresentado na seção 2.
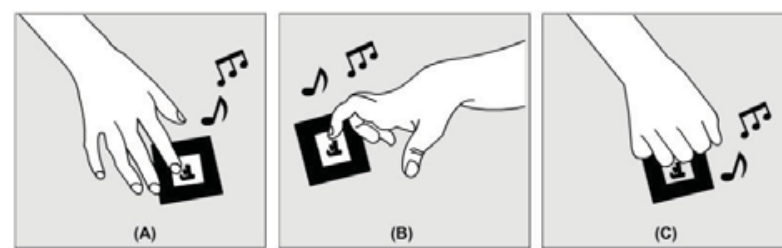

Figura 4: Interação com os cartões musicais

O timbre do piano é o timbre padrão das notas musicais. Mas, pode-se alterar o timbre das notas musicais, colocando-se no campo de visão da webcam, o cartão com o desenho do instrumento musical desejado. Não é necessário permanecer com o cartão do instrumento musical (corda ou sopro) no campo de visão da webcam. Uma vez que o cartão do instrumento for reconhecido pelo sistema, todas as notas musicais têm seu timbre alterado automaticamente a partir de então (Figura 5).

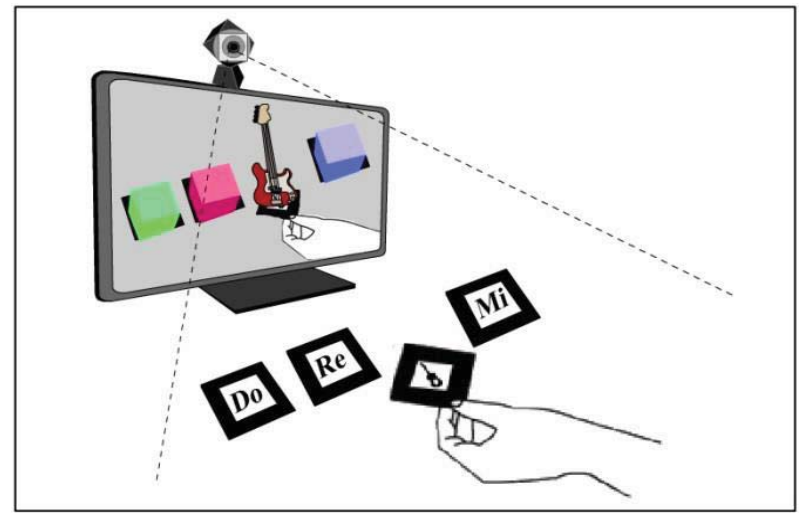

Figura 5: Mudando o timbre das notas musicais.

Os sons dos instrumentos musicais com altura definida (instrumentos de corda e sopro) são utilizados apenas para trocar o timbre das notas musicais. Já, no caso dos instrumentos de percussão (de altura não definida (ou indeterminada), é necessário utilizar o próprio cartão do referido instrumento para sua execução (Figura 6).

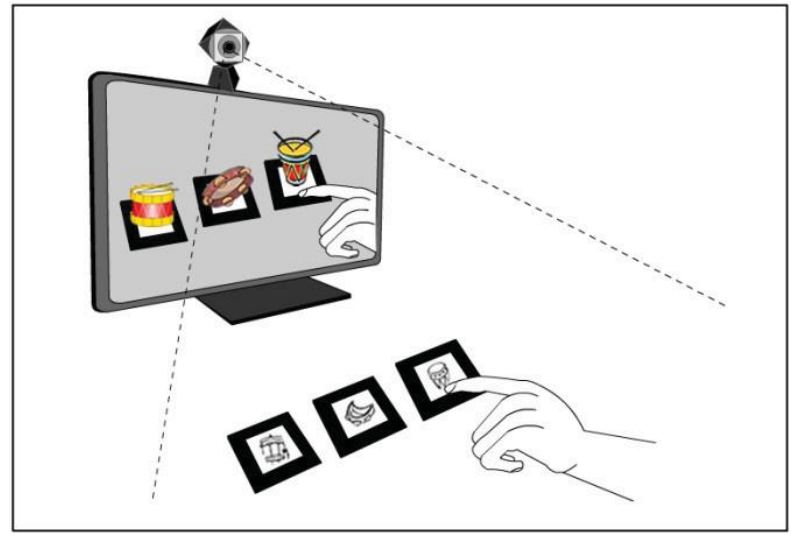

Figura 6: Instrumentos musicais de percussão.
O GenVirtual também possui um recurso para auxiliar nas atividades de recriação musical. Foram criadas partituras compostas por melodias que podem ser lidas por meio de bolinhas coloridas. As cores das notas musicais da partitura correspondem às mesmas cores das notas musicais do GenVirtual. A Figura 7 mostra uma das partituras musicais disponível no GenVirtual retirada do livro "Canções no Piano" baseado na obra Winnie the Pooh da Companhia Disney Enterprise.

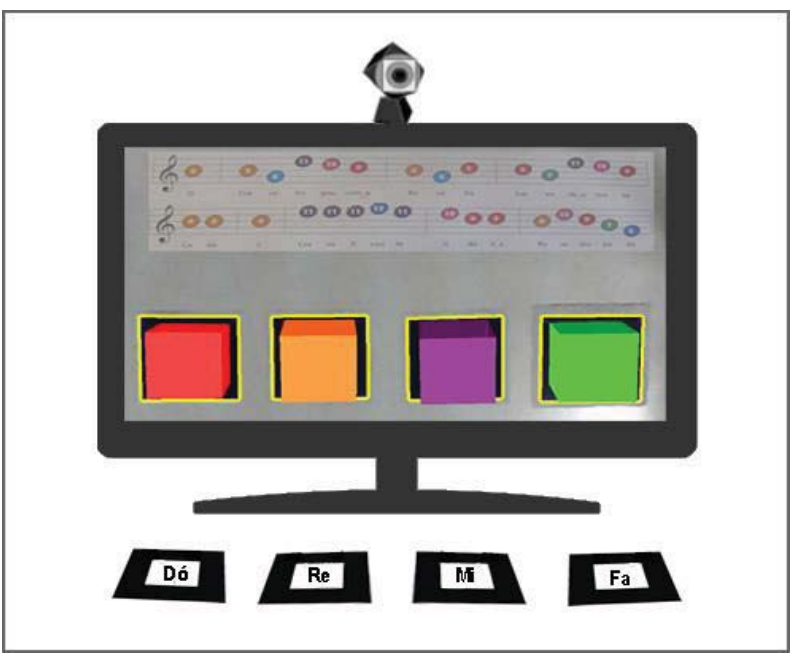

Figura 7: PartituraMusical do GenVirtual

Além da composição livre, o GenVirtual contém um jogo de memória musical baseado em sons e cores inspirado no jogo Genius fabricado pela empresa Estrela, por isso foi denominado inicialmente de "Genius Musical Virtual" e depois de GenVirtual. A Tabela 1 mostra as principais diferenças entre o jogo Genius da Estrela e o jogo de memória musical do GenVirtual.

\begin{tabular}{|c|c|}
\hline $\begin{array}{l}\text { GENIUS } \\
\text { DA ESTRELA }\end{array}$ & $\begin{array}{c}\text { JOGO DE MEMÓRIA MUSICAL } \\
\text { DO GENVIRTUAL }\end{array}$ \\
\hline $\begin{array}{l}\text { Possui } 4 \text { botões } \\
\text { coloridos. }\end{array}$ & $\begin{array}{l}\text { Possibilidade de adicio- } \\
\text { nar ou remover elementos } \\
\text { virtuais (botões). }\end{array}$ \\
\hline $\begin{array}{l}\text { Botões em posições } \\
\text { fixas em formato } \\
\text { de disco voador. }\end{array}$ & $\begin{array}{l}\text { Possibilidade de utilizar } \\
\text { os cartões marcadores em } \\
\text { diferentes tamanhos e em } \\
\text { diferentes posicionamen- } \\
\text { tos. }\end{array}$ \\
\hline Sons eletrônicos. & $\begin{array}{l}\text { Sons de notas musicais } \\
\text { (Dó, Ré, Mi, Fá, Sol, Lá } \\
\text { e Si) e de instrumentos } \\
\text { de percussão. }\end{array}$ \\
\hline Sons invariantes. & $\begin{array}{l}\text { Possibilidade de alterar } \\
\text { o timbre dos sons (piano, } \\
\text { guitarra, violão, flauta, } \\
\text { trompete e trombone) além } \\
\text { da possibilidade de alte- } \\
\text { rar a escala musical. }\end{array}$ \\
\hline $\begin{array}{l}\text { Sequência sonora } \\
\text { randômica. }\end{array}$ & $\begin{array}{l}\text { Possibilidade de criar } \\
\text { sequência sonora ou musi- } \\
\text { cal. }\end{array}$ \\
\hline
\end{tabular}

Tabela 1: Genius Estrela x Jogo de Memória Musical do GenVirtual 
Os cartões musicais do jogo são definidos como "peças". Com estas peças, pode-se aumentar ou diminuir o processamento de estímulos vindos da interface do jogo. Em seu funcionamento, o sistema gera uma sequência musical composta de notas musicais. O usuário pode escolher se a sequência musical deve ser gerada aleatoriamente, por meio do sorteio das notas, ou se deve ser gerada com base em alguma melodia gravada no sistema. No primeiro caso, o usuário deve apenas informar o tamanho da sequência musical, isso implica em determinar o número de rodadas do jogo. No segundo caso, o usuário escolhe a melodia que deve compor a sequência musical e o sistema informa os cartões musicais necessários para o jogo e a quantidade de rodadas.

Inicialmente, o sistema dispara uma animação no elemento virtual 3D (Figuras 8 e 9), referente ao primeiro elemento da sequência musical. O elemento virtual emite seu som correspondente e fica a espera da interação do usuário (Figura 8b). Quando o usuário obstrui um cartão, o sistema executa o som correspondente e verifica se o cartão obstruído corresponde ao primeiro elemento da sequência musical. Caso negativo, o sistema emite uma mensagem de erro e o jogo é finalizado. Caso positivo, o sistema atualiza a pontuação do jogo e verifica se a sequência musical chegou ao final. Se sim, então o sistema finaliza o Jogo e informa que o usuário venceu. Se não, o sistema incrementa a sequência musical e inicia o novo ciclo, aumentando assim, o desafio de memória e retenção de informação do jogo.

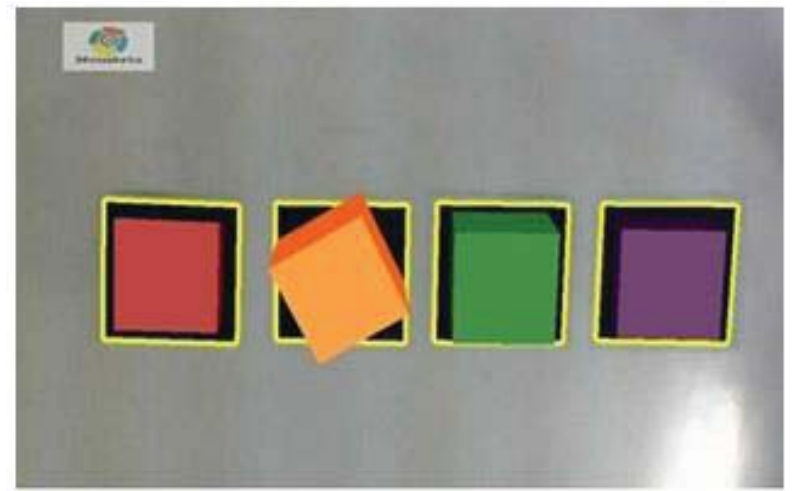

Figura 8: Animação do jogo da memória musical do GenVirtual

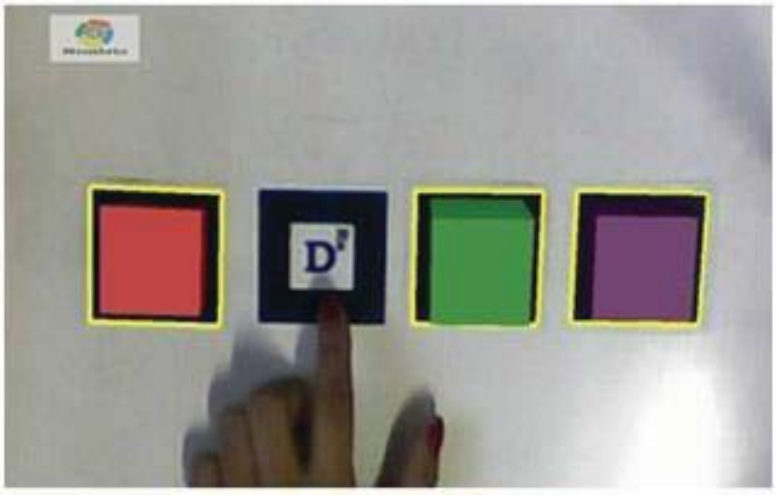

Figura 9: Interação do usuário com o jogo memória musical

O diferencial do GenVirtual está na possibilidade de permitir ao terapeuta realizar o planejamento da tarefa de cada indivíduo, dependendo de suas limitações físicas e cognitivas. Isto ocorre através da quantidade e disposição dos cartões marcadores sobre a mesa. De acordo com os especialistas em Educação Musical, é importante ter a referência do movimento para que seja possível controlar a motricidade do indivíduo, caso contrário, não ocorrerá o aprendizado motor. Por meio da interação com os objetos virtuais é possível também estimular habilidades cognitivas, tais como: concentração, memorização, percepção visual e auditiva, além da aprendizagem musical em si.

\subsection{Propostas de Atividades Musicais}

As atividades musicais podem desenvolver-se de acordo com várias abordagens e métodos, sendo os mais comuns: improvisação; recriação e composição musical; audição sonora e musical [44], [45], [46], [47]. A Tabela 2, descreve como estas atividades podem ser desenvolvidas com uso do GenVirtual. Essa análise foi feita durante uma avaliação preliminar do GenVirtual, ainda na fase de desenvolvimento, com especialistas em musicoterapia.

\begin{tabular}{|c|c|}
\hline Ativ. & Descrição \\
\hline 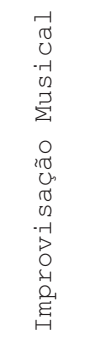 & $\begin{array}{l}\text { Executar, por meio dos cartões musi- } \\
\text { cais, os elementos sonoros (notas } \\
\text { musicais e instrumentos musicais), de } \\
\text { improviso, sozinho, em dueto ou em } \\
\text { grupo. os resultados desta prática } \\
\text { poderão ser mensurados por meio da } \\
\text { improvisação, onde o indivíduo é esti- } \\
\text { mulado a pensar e raciocinar sozinho } \\
\text { fazendo uma reflexão quanto às formas } \\
\text { de assimilar e transmitir sua expres- } \\
\text { são musical. }\end{array}$ \\
\hline 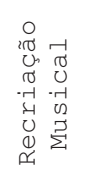 & $\begin{array}{l}\text { Executar, reproduzir, transformar ou } \\
\text { interpretar uma música ou parte dela } \\
\text { por meio dos cartões musicais (notas } \\
\text { musicais e instrumentos musicais). A } \\
\text { recriação poderá ser gravada como um } \\
\text { registro para posterior avaliação. }\end{array}$ \\
\hline
\end{tabular}




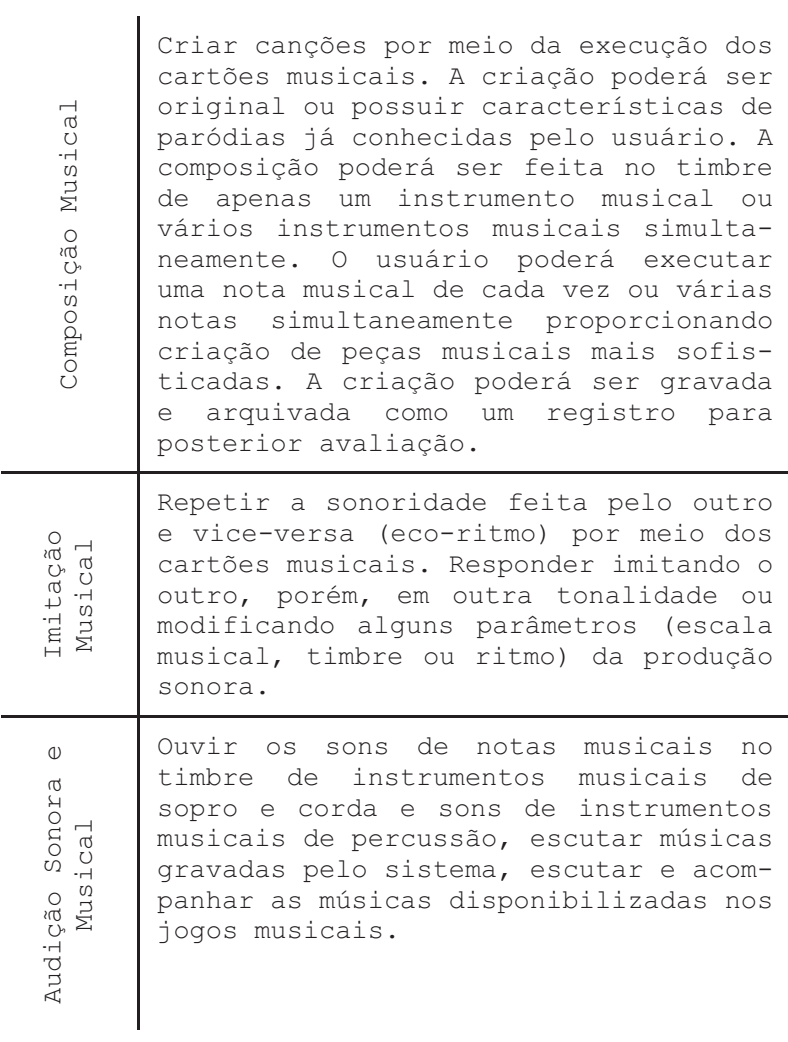

Tabela 2: Propostas de atividades musicais apoiadas pelo GenVirtual

\section{Avaliações do GenVirtual}

O GenVirtual foi avaliado em duas situações de uso: a) por um grupo de especialistas em música com vistas a validar o conjunto de requisitos relacionados ao "fazer musical" de pessoas com deficiência; b) por um grupo de crianças com déficits motores e cognitivos em sessões de Musicoterapia na Associação de Assistência à Criança Deficiente (AACD); A metodologia e os resultados destas avaliações são descritos a seguir.

\subsection{Avaliação do "fazer musical"}

O objetivo deste estudo foi validar os requisitos referentes ao "fazer musical" de pessoas com deficiência com base nas atividades musicais propostas na Tabela 1 . O estudo foi realizado no setor de Musicoterapia da AACD durante um encontro nacional de musicoterapeutas. Participou desta avaliação um grupo composto por 11 musicoterapeutas advindos de diferentes estados brasileiros. Os materiais utilizados foram: o software GenVirtual instalado em um computador (Pentium II Intel de $1.0 \mathrm{GHz}$ e $512 \mathrm{mb}$ de memória RAM), um monitor de 15", uma webcam (Logitech Webcam Pro 9000) equipada com um tripé e caixas acústicas. A coleta de dados foi feita por meio de um questionário (Tabela 3) elaborado pelos autores.

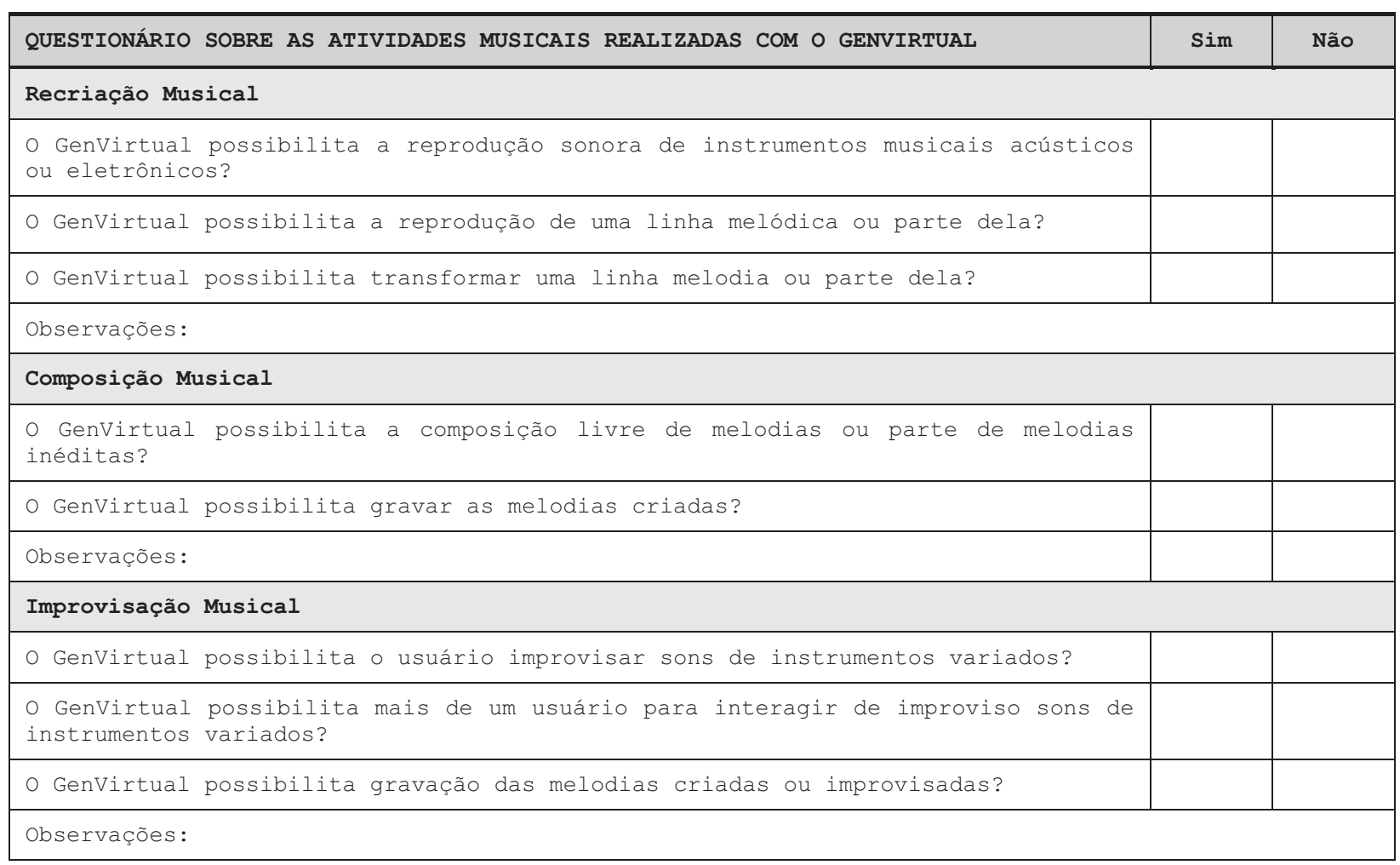




\begin{tabular}{|l|l|l|}
\hline \multicolumn{2}{|l|}{ Audição sonora e Musical } \\
\hline O GenVirtual possibilita a reprodução do áudio do material sonoro gravado? & \\
\hline $\begin{array}{l}\text { As sonoridades dos instrumentos musicais do GenVirtual se aproximam da sonorida- } \\
\text { de dos instrumentos convencionais? }\end{array}$ & & \\
\hline Observações: & & \\
\hline
\end{tabular}

Tabela 3: Questionário sobre as atividades realizadas com o GenVirtual aplicado no Encontro de Musicoterapeutas

A musicoterapeuta, responsável pelo setor, formou grupos compostos por três pessoas. Cada grupo realizou oficinas de uso do GenVirtual. Inicialmente, o sistema foi apresentado aos participantes que, em seguida, interagiram com o mesmo. Durante o experimento, a musicoterapeuta apontou possíveis atividades de musicoterapia que poderiam ser apoiadas com uso do sistema e solicitou que os musicoterapeutas testassem estas atividades. Ao final do experimento foi solicitado, a cada um dos participantes, que respondessem o questionário. Após a análise dos dados coletados nos questionários, constatou-se que o GenVirtual contempla todas as atividades musicais propostas na Tabela 1:

- A recriação musical pode ser realizada por meio da interação com os cartões musicais. Pode-se recriar composições escritas nas partituras do GenVirtual.

- Pode-se transformar uma linha melódica ou parte dela alterando os instrumentos musicais disponíveis no sistema.

- A composição musical pode ser realizada por meio da interação com os cartões musicais. Pode-se criar composições livres ou parte de melodias inéditas e gravá-las no sistema.

- A audição sonora e musical é possibilitada pelo recurso do gravador e da clave de sol disponíveis no sistema.

- A imitação musical pode ser realizada por meio da interação com os cartões musicais onde o usuário pode interagir de improviso, sons de instrumentos musicais variados. As sonoridades dos instrumentos musicais da ferramenta se aproximam da sonoridade dos instrumentos convencionais.

Além das questões objetivas, foram feitas duas perguntas abertas em relação aos pontos fortes e fracos do GenVirtual. Os pontos fortes destacados são:

- Possibilidade de se trabalhar os sons das notas musicais relacionadas às cores.

- $\quad$ Facilidade e simplicidade de uso da aplicação.
- Software livre (GenVirtual está disponível para download gratuito no endereço eletrônico www.anagrasi.com.br).

- Apresenta um recurso moderno e atual que agrada, entretém e diverte as crianças desta nova geração.

- Possibilidade de usar o computador com o paciente.

- Possibilidade de uso por parte de pessoas com graves comprometimentos motores.

- Possibilidade de criar acordes por meio da execução de dois ou mais cartões musicais simultaneamente, o que possibilita estimular o uso de ambas as mãos.

- Possibilidade de gravar e reproduzir improvisos musicais.

Os pontos fracos destacados foram:

- Sonoridade de alguns instrumentos musicais um pouco diferente do instrumento convencional, o que pode atrapalhar as atividades de apreciação sonora e musical.

- Resultado sonoro, às vezes, é "lentificado" pelo sistema, ou seja, a duração dos sons não varia, pois já é pré-definido pelo sistema.

\subsection{Avaliações de Uso em Musicoterapia}

O objetivo deste estudo foi avaliar a motivação e a satisfação dos usuários (crianças com deficiência e seus cuidadores) em intervenções de Musicoterapia apoiadas pelo GenVirtual. O estudo foi realizado no setor de Musicoterapia da AACD. Os materiais utilizados foram os mesmos já citados no estudo anterior (seção 5.2). Este estudo foi aprovado pelo Comitê de Ética em Pesquisa da AACD com o protocolo $\mathrm{N}^{\circ}$ protocolo $\mathrm{n}^{\circ} 089 / 10$.

Participaram deste estudo três pacientes enquadrados no programa de reabilitação para membros superiores (MMSS) no setor de Musicoterapia da AACD com média de 6 anos de idade. Foram excluídos do estudo os indivíduos com baixa cognição, com intercorrências clínicas e com baixa aderência ao tratamento de reabilitação.

A coleta de dados foi feita por meio de um questioná- 
rio semiestruturado de satisfação da interação do usuário baseado no método QUIS (Questionnaire for User Interface Satisfaction). Um questionário de pré-teste foi aplicado antes do início da sessão para coletar o perfil dos usuários (paciente e terapeuta). Outro questionário de pós-teste (Tabela 4) foi aplicado no terapeuta após cada sessão. O questionário de pós-teste buscou coletar dados referentes à: 1) satisfação do terapeuta; 2) motivação dos pacientes; 3) facilidade de uso 4) aplicabilidade para o setor; 5) interesse em continuar utilizando o GenVirtual; 6) Pontos fortes e fracos da ferramenta. Foram elencados três procedimentos para estimular a função da musicalidade e a função motora com os seguintes objetivos: estímulo da percepção visual; estímulo da percepção auditiva; e imitação motora a partir do feedback auditivo.

\section{QUESTIONÁRIO DA SATISFAÇÃO DA INTERAÇÃO DO USUÁRIO}

\begin{tabular}{|c|c|}
\hline 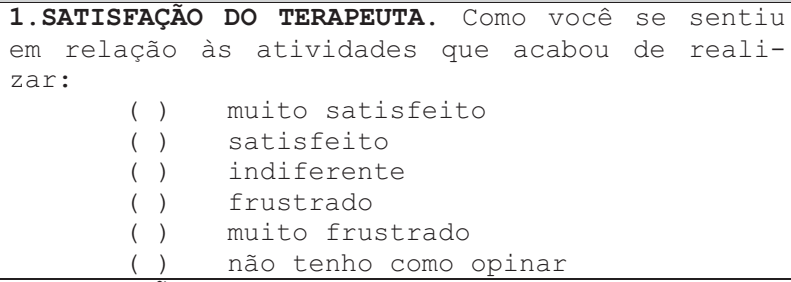 & $\begin{array}{l}\text { 6. CoNTINUIDADE DE USO. Você gostaria de utili- } \\
\text { zar o GenVirtual para reabilitação de outro(s) } \\
\text { paciente(s): } \\
\qquad \begin{array}{ll}(\text { ) } & \text { sempre } \\
(\text { ) às vezes } & \\
(\text { ) indiferente } \\
(\text { ) quase nunca } \\
(\text { ) nunca } \\
(\text { ) não tenho como opinar }\end{array}\end{array}$ \\
\hline $\begin{array}{l}\text { 2.MOTIVAÇÃo DO PACIENTE. Como seu paciente se } \\
\text { sentiu em relação às atividades que acabou de } \\
\text { realizar: } \\
\text { ( ) muito motivado } \\
\text { ( ) motivado } \\
\text { ( ) indiferente } \\
\text { ( ) desmotivado } \\
\text { ( ) muito desmotivado } \\
\text { ( ) não tenho como opinar }\end{array}$ & $\begin{array}{l}\text { 7. PONTOS FORTES. Liste os pontos fortes sobre } \\
\text { a atividade que acabou de realizar: }\end{array}$ \\
\hline $\begin{array}{l}\text { 3.FACILIDADE } \text { DE USO. Utilizar } \circ \text { GenVirtual com } \\
\text { seu paciente foi: } \\
\qquad \begin{array}{ll}\text { ( ) } & \text { muito fácil } \\
\text { ( ) } & \text { fácil } \\
\text { ( ) } & \text { indiferente } \\
\text { ( ) } & \text { muito difícil } \\
\text { ( ) não tenho como opinar }\end{array}\end{array}$ & $\begin{array}{l}\text { 8. PONTOS FRACOS. Liste os pontos fracos sobre } \\
\text { a atividade que acabou de realizar: }\end{array}$ \\
\hline $\begin{array}{l}\text { 4. APLICABILIDADE PARA O SETOR. Como você consi- } \\
\text { dera as possibilidades de uso do GenVirtual } \\
\text { para apoiar os métodos e procedimentos musico- } \\
\text { terapêuticos: } \\
\qquad \begin{array}{l}\text { ( ) muito adequado } \\
\text { ( ) adequado } \\
\text { ( ) indiferente } \\
\text { ( ) inadequado } \\
\text { ( ) muito inadequado } \\
\text { () não tenho como opinar }\end{array}\end{array}$ & $\begin{array}{l}\text { 9. Você realizou alguma(s) atividade(s) que } \\
\text { anteriormente não era (m) possível(s) de ser(em) } \\
\text { feita(s) sem uso do computador? }\end{array}$ \\
\hline $\begin{array}{l}\text { 5. CONTINUIDADE DE USO. Você gostaria de conti- } \\
\text { nuar utilizando o GenVirtual com este paciente: } \\
\text { ( ) sempre } \\
\text { ( ) às vezes } \\
\text { ( ) indiferente } \\
\text { ( ) quase nunca } \\
\text { ( ) nunca } \\
\text { ( ) não tenho como opinar }\end{array}$ & $\begin{array}{l}\text { 10. Deixe suas sugestões e comentários a res- } \\
\text { peito da(s) atividade(s) que acabou de reali- } \\
\text { zar: }\end{array}$ \\
\hline
\end{tabular}

Tabela 4: Questionário de satisfação da interação do usuário aplicado no terapeuta na AACD

Os pacientes e seus cuidadores foram informados sobre os objetivos e os procedimentos da pesquisa. Foi solicitado aos cuidadores que preenchessem o Termo de Consentimento Livre e Esclarecido para que os experimentos pudessem ser registrados através de fotos e vídeos. Os testes foram feitos individualmente, na sala de musicoterapia, e com duração de 30 minutos cada. O participante foi adequadamente posicionado sentado, em frente a uma mesa, sobre a qual estavam os materiais.

Procedimentos: 


\section{- Percepção visual:}

- O terapeuta cita uma sequência de cores para que o paciente possa reproduzi-la com o GenVirtual.

\section{- Percepção sonora:}

- O terapeuta executa uma nota musical em algum instrumento musical e o paciente tenta executar a mesma nota com o GenVirtual.

\section{- Imitação musical:}

- O terapeuta deve executar uma determinada sequência musical com o teclado musical para que o paciente possa reproduzi-la com o GenVirtual.

- O paciente deve executar uma sequência musical com o GenVirtual e o terapeuta deve reproduzi-la com o teclado musical.

As Figuras 10 e 11 mostram imagens dos testes realizados com os pacientes e musicoterapeuta no setor de musicoterapia da AACD.

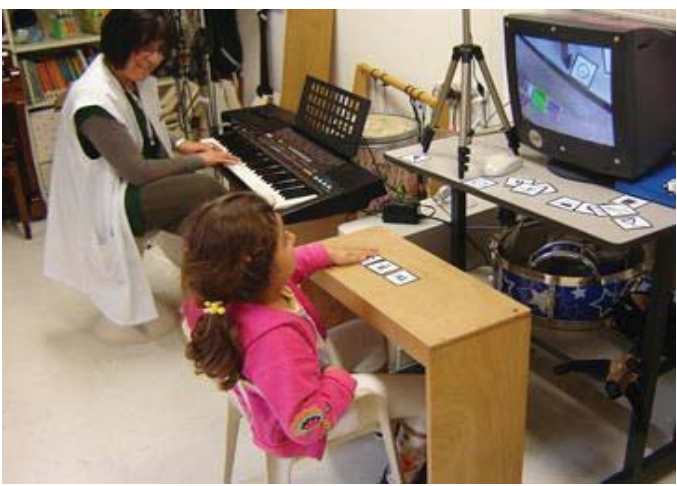

Figura 10: Paciente C.M. usando o GenVirtual

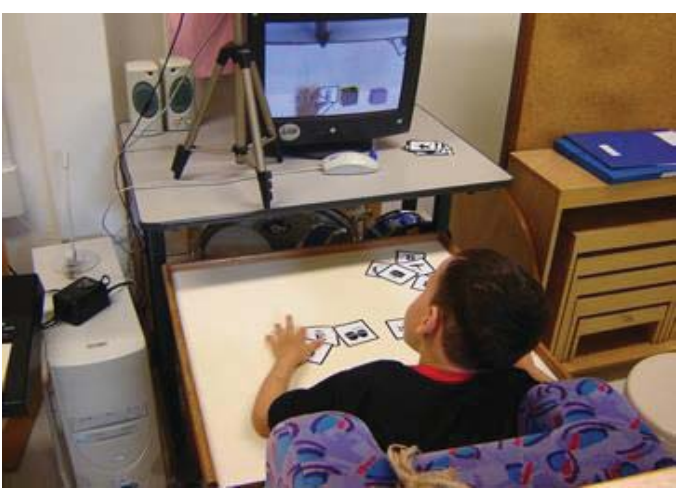

Figura 11: Paciente H.Y. utilizando o GenVirtual.

Os resultados da análise dos dados coletados nos questionários indicaram que:
- O terapeuta ficou satisfeito com os resultados das intervenções e manifestou interesse em continuar com o uso da ferramenta tanto com estes pacientes quanto com outros que porventura venham necessitar do mesmo plano de terapia.

- O paciente se sentiu muito motivado após as intervenções. Além das atividades solicitadas pelo terapeuta, o paciente mostrou interesse em continuar explorando a ferramenta, tentando configurar novos layouts com os cartões e reproduzindo sons em escalas diferentes.

- Tanto o terapeuta, quanto os pacientes e cuidadores que assistiram a sessão atestaram ser fácil de usar e de aprender a usar.

- Segundo o terapeuta, o GenVirtual possui aplicabilidade adequada para o setor de Musicoterapia tanto para atividades de reabilitação motora quanto cognitiva.

- Segundo o terapeuta, o GenVirtual pode facilitar as atividades musicais das crianças com o diagnóstico de distrofia muscular que, para estes casos, não são recomendadas atividades que possam promover a fadiga muscular ou perdas energéticas. A interação com o GenVirtual, ou seja, o disparo do som planejado é feita por meio do mínimo movimento da mão, detectado através dos cartões marcadores, sem necessidade de percuti-los como ocorre na interação com os instrumentos musicais naturais.

- Os cuidadores manifestaram interesse em ter acesso ao GenVirtual em domicílio.

Os pontos fortes destacados foram:

- Diversão: a possibilidade de acompanhar o ritmo musical da terapeuta e de criar sua própria música despertou o interesse e a diversão do paciente. De acordo com [48], a diversão é um aspecto contribuinte para o prazer e inclui atenção, concentração, envolvimento, entre outros aspectos.

- Motivação: o GenVirtual melhorou o desempenho e a ação do paciente ao realizar os exercícios de forma diferente possibilitando sair da rotina dos exercícios convencionais.

- Criatividade: foi observado que alguns pacientes demonstraram suas habilidades musicais, o que possibilita considerar que o GenVirtual pode ser utilizado como meio incentivador de criatividade. 
- Utilidade: o GenVirtual possibilitou o desenvolvimento de diversas atividades terapêuticas feitas na forma a partir de um planejamento.

- Aprendizado: as tarefas foram tanto por parte do terapeuta, no momento de criar o planejamento das tarefas, quanto por parte do paciente ao interagir com o sistema, de forma natural dentro de um processo dedutivo de fácil realização.

- Inclusão Digital: nenhum dos pacientes havia anteriormente interagido com um ambiente de Realidade Aumentada.

- Software livre: pode ser utilizado em domicílio sem custo para aquisição.

Os pontos fracos destacados foram:

- Sonoridade pobre de alguns instrumentos musicais, pois os sons são produzidos pelo sintetizador do computador.

- A duração e a intensidade dos sons são prédefinidas pela ferramenta, não sendo possível alterá-las.

\section{Conclusões e Perspectivas Futuras}

Este capítulo apresentou detalhes do projeto e implementação de uma interface musical com realidade aumentada para apoiar o "fazer musical" de pessoas com deficiência. O sistema foi desenvolvido em etapas conforme os requisitos iam sendo elencados a partir dos testes funcionais e de usabilidade de uso da ferramenta.

As avaliações permitiram confirmar que o GenVirtual possibilitou o desenvolvimento das atividades musicais propostas na Tabela 1 (improvisação, recriação e composição musical, audição sonora e musical). Os dados coletados por meio de questionários semiestruturados mostraram que: a) as tarefas foram realizadas dentro do esperado por parte dos usuários, o que torna o GenVirtual uma ferramenta fácil de usar e fácil de aprender a usar; b) o GenVirtual melhorou o desempenho e o estímulo da ação dos pacientes, características estas que contribuem para a motivação durante a terapia; c) o terapeuta se sentiu satisfeito com uso do GenVirtual e mostrou interesse em continuar a usá-lo com outros pacientes em outras intervenções; d) na visão do terapeuta, os pacientes se mostram mais motivados e interessados, passando a perceber a terapia como divertida; e) muitos pacientes se dispuseram à mostrar as habilidades musicais, fora do tempo estipulado para a terapia, o que indica que o GenVirtual pode ser considerado um instrumento incentivador de criatividade; f) proporcionou a inclusão digital, visto que nenhum dos pacientes envolvidos nos testes tinham interagido anteriormente com tecnologias de Realidade $\mathrm{Au}-$ mentada.

$\mathrm{O}$ fato dos testes e avaliações terem sido feitos dentro de centros de reabilitação favoreceram o envolvimento de outros setores da reabilitação como a Terapia Ocupacional. Avaliações com pessoas com Distrofia Muscular de Duchenne estão sendo realizadas para novas constatações da ferramenta.

Como trabalhos futuros, pretende-se continuar a implementação de novos recursos no GenVirtual, a fim de viabilizar seu uso em atividade não-assistidas (sem a presença do especialista), seja em ambiente escolar, hospitalar ou clínica, seja em ambiente domiciliar. Para isso, será necessário um estudo detalhado sobre estratégias, informações relevantes e mecanismos para medição e registro de informações durante as atividades para acompanhamento e análise pelos especialistas.

Dado que se baseia em plataforma computacional convencional (computador com webcam), o GenVirtual, já está em condições de ser utilizado em centros de reabilitação, clínicas e ambiente domiciliar. O GenVirtual está disponível para download gratuito no endereço eletrônico www.anagrasi.com.br.

\section{Referências}

[1] T.A. Brito Por uma educação musical do pensamento: educação musical menor. Revista da Associação Brasileira de Educação Musical (ABEM), 21(1):25-34, 2009.

[2] T.A. Brito Gesto/ação/pensamento musical: o fazer musical da infância. In Anais do XIV Encontro Anual da Associação Brasileira de Educação Musical (ABEM), Belo Horizonte, 2005.

[3] J.O. Oliveira, N. Cavalari. A Musicalidade Infantil aliada a Psicomotricidade. Caderno Multidisciplinar de Pós-Graduação da UCP. Pitanga. 1(1):195-205, 2010.

[4] C.E. Souza e M.C.L. Joly. A Importância do Ensino Musical na Educação Infantil. Cadernos da Pedagogia. São Carlos, 4(4): 96-110, 2010.

[5] D.B.S. Bündchen. A Relação Ritmo-Movimento no Fazer Musical Criativo: uma abordagem construtivista na prática de canto oral. Dissertação de Mestrado do curso de Educação da Universidade do Rio Grande do Sul, 2005.

[6] E.C. Menezes. Convivendo, conversando, criando e fazendo música: a educação musical no 
Corpo Cidadão. Revista da Associação Brasileira de Educação Musical (ABEM), 20(27):43-54, 2012.

[7] J.D. Silva Jr. A Utilização da Música com Objetivos Terapêuticos: interfaces com a bioética. Dissertação de Mestrado apresentado ao Programa de Pós-Graduação em Música da Escola de Música e Artes Cênicas da Universidade Federal de Goiás, 2008.

[8] A.C.C. Paiva, M.B. Zagonel, M.N. V. Arouck, S.T. Silva. A Música como Recurso para a Aprendizagem do Aluno Hiperativo: relato de uma experiência. In XVI Encontro Anual da Associação Brasileira de Educação Musical, 2007.

[9] L. Paula, M.H.J. Borges. O Ensino da Performance Musical: uma abordagem teórica sobre o desenvolvimento dos eventos mentais relacionados às ações e emoções presentes no Fazer Musical. Revista Música Hodie, 4(1):29-44, 2004.

[10] R. Fried Integrating music in breathing training and relaxation: I. Background, rationale, and relevant elements. Biofeedback Self Regul, 15(1):161-9, 1990.

[11] S. Ray, X. Andreola. O Alongamento Muscular no Cotidiano do Performer Musical: estudos, conceitos e aplicações. Revista Música Holdie, 5(1):21-34, 2005.

[12] M. Franco, A.B. RodriguesB. A música no alívio da dor em pacientes oncológicos. Einstein: São Paulo, 7(2): 147-151, 2009.

[13] R. Chaffim. Thinking about performance: memory, attention and practice. In International Symposium on Performance Science, pp. 689699, 2011.

[14] L. Jäncke. Music, memory and emotion. Journal of Biology, 7(21):11-21, 2008.

[15] C. Pacchetti, et al. Active music therapy in parkinson's disease: an integrative method for motor and emotional rehabilitation. Psychosomatic Medicine: Journal of Biobehavioral Medicine, 62(3): 386-393, 2000.

[16] S.L. Siedliecki, M. Good. Effect of music on power, pain, depression and disability. Journal of Advanced Nursing, 54(1): 553-562, 2006.

[17] S.B. Hanser, L.W. Thompson. Effects of a music therapy strategy on depressed older adults. Journal of Gerontology, 49(6): 265-269, 1994.

[18] V.S. Louro, C.Y. Ikuta, M. Nascimento. Música e deficiência: levantamento de adaptações para o fazer musical de pessoas com deficiência. Arquivos Brasileiros de Paralisia Cerebral, 1(2):11$17,2005$.

[19] V.S. Louro. Educação musical e musicoterapia: adaptações do fazer musical em prol da aprendizagem ou reabilitação de pessoas com deficiência. In: M. Nascimento. Musicoterapia e a reabilitação do paciente neurológico. São Paulo: Memmon, pp. 398-410, 2009.

[20] L. Soares. Programa de apoio pedagógico e inclusão: um estudo de caso. Revista da Associação Brasileira de Educação Musical (ABEM), 20(27): 55-64, 2012.

[21] CETIC - Centro de Estudos sobre Tecnologias da Informação e Comunicação. Núcleo de Informação e Coordenação do Ponto BR: São Paulo, 2012.

[22] R.D. Lopes. et al. O uso do computador e da internet em escola públicas de capitais brasileiras. In: Fundação Victor Civita: Estudos \& Pesquisas Educacionais. Fundação Victor Civita: São Paulo, 2010. pp.275-34.

[23] V. Mattozo, E. Specialski O Ciberespaço e as Redes de Computadores na Construção do Novo Conhecimento. Revista Brasileira de Informática na Educação (RBIE), 6(1):1-10, 2000.

[24] M. Struchiner, T.R. Gianella. Análise do Processo de Integração de Informação e Comunicação em Atividades Educativas no Ensino Fundamental no Contexto do "Programa Um Computador por Aluno". In F.F. Sampaio, M.F. Elia (Org.) Projeto um computador por aluno: pesquisas e perspectivas, Rio de Janeiro: NCE/UFRJ, 2012.

[25] G.A. Yatsuda, et al. TAW - Treinamento Auditivo pela Web: ensino musical a distância. Anais do XXII Simpósio Brasileiro de Informática na Educação, 2011, pp. 771-780.

[26] A.J. Martins, C. Fiolhais, J. Paiva. Simulações On-line no Ensino da Física e da Química. Revista Brasileira de Informática na Educação (RBIE), 11(1): 1-10, 2003.

[27] M. Gorman, et al. A camera-based musicmaking tool for physical rehabilitation. Computer Music Journal, 31(2):39-53, 2007.

[28] P. Ellis, The Music of Sound: a new approach for children with severe and profound and multiple learning difficulties, in the British Journal of Music Education, 14(2):17-186, 1997. 
[29] E. Streeter, et al. Computer aided music therapy evaluation: testing the music therapy Logbook prototype 1 system. The Arts in Psychotherapy 39(1):1-10, 2012.

[30] O. Shaer, E. Hornecker. Tangible User Interfaces: Past, Present and Future Directions. Foundations and Trends in Human-Computer Interaction, 3(2):1-137, 2010.

[31] H. Ishii. The Tangible User Interface and Its Evolution. Communications of the ACM, 51(6): 32-36, 2008.

[32] E. Shahar, E. SoundStrand: a Tangible Interface for Composing Music with Limited Degrees of Freedom. Dissertação de mestrado apresentada à Faculdade de Arquitetura e Planejamento do Instituto Tecnológico de Massachusetts, 2012.

[33] M. Zajc, A.I. Starcic. Potentials of the Tangible User Interface (TUI) in Enhancing Inclusion of People with Special Needs. In: ICT-Assisted Learning and e-Accessibility, 7327(1): 261-270, 2012.

[34] B. Schiettecatte, J. Vanderdonckt, AudioCubes: a Distributed Cube Tangible Interface Based. Proceedings of the Second International Conference on Tangible and Embedded Interaction, 2008, pp. 3-10.

[35] S. Jorda, M. Kaltenbrunner, G. Geiger, R. Becina. The Reactable. Proceedings of the International Computer Music Conference (ICMC), 2005.

[36] M. Kaltenbrunner, R. Bencina. reacTIVision: a computer-vision framework for table based tangible interaction. Proceedings of the 1st international conference on Tangible and embedded interaction, 2007, pp. 69-74.

[37] L. Garber. Tangible User Interfaces: technology you can touch. IEEE Computer, 45(6):15-18, 2012.

[38] L. Villafuerte, M.S. Markova, S. Jordà. Acquisition of Social Abilities Through Musical Tangible User Interface: children with autismo spectrum condition and the Reactable. ACM SIGCHI Conference on Human Factors in Computing System, pp. 745-760, 2012.

[39] E. Costanza, S.B. Shelley, J. Robinson. Introducing Audio D-Touch: a tangible user interface for music composition and performance. Anais do International Conference on Digital Audio Effects, pp.8-11, 2003.
[40] S. Suzuki, S. Nurtured by Love. A New Approach to Education (Waltraud Suzuki, Trans.). New York: Exposition Press, 1969.

[41] R. Berry, M. Makino, N. Hikawa, M. Suzuki. The Augmented Composer Project: The Music Table. International Symposium on Mixed and Augmented Reality, Japão, pp. 338-339, 2003.

[42] C. Kirner, T.G. Kirner, Virtual Reality and Augmented Reality Applied to Simulation Visualization. In: El Sheikh, A.A.R.; Al Ajeeli, A.; Abu-Taieh, E.M.O. (Ed.). Simulation and Modeling: Current Technologies and Applications. 1 ed. Hershey-NY: IGI Publishing, 1(1): 391-419, 2008 .

[43] R. Azuma, et al. Recent Advances in Augmented Reality. IEEE Computer Graphics and Applications, 21(6):34-47, 2001.

[44] A. Baranow. Musicoterapia: uma visão geral. Rio de Janeiro: Enelivros, 1999.

[45] K.E. Bruscia. Definindo musicoterapia. 2. ed. Rio de Janeiro: Enelivros, 2000.

[46] M. Nascimento. Musicoterapia: princípio e prática. In: A.C. Fernandes, et al. AACD medicina e reabilitação: princípio e prática. São Paulo: Artes Médicas, 2006. p. 853-864.

[47] C. Ikuta. Métodos de intervenção Musicoterapêutica e suas aplicações. In: M. Nascimento. Musicoterapia e a reabilitação do paciente neurológico. São Paulo: Memmon, 2009. p. 82-101.

[48] J. Preece, Y. Rogers, H. Sharp. Design de interação - além da interação homem-computador. São Paulo: Bookman Coahia Editora, p.348, 2005. 